\title{
Nucleosynthetic W-Mo isotope variations in Solar System materials
}

\author{
G. BudDE ${ }^{* 1,2}$, F. TISSOT $^{2}$, C. BURKHARDT ${ }^{1}$, T. KLEINE $^{1}$
}

${ }^{1}$ Institut für Planetologie, University of Münster, WilhelmKlemm-Straße 10, 48149 Münster, Germany

${ }^{2}$ The Isotoparium, Division of Geological and Planetary Sciences, California Institute of Technology, Pasadena, CA 91125, USA (*correspondence: budde@caltech.edu)

Nucleosynthetic isotope variations are a powerful tool for investigating the origin and early evolution of the Solar System. For instance, Mo isotopes can clearly distinguish between different nucleosynthetic processes and thus revealed a fundamental dichotomy between inner (non-carbonaceous, $N C$ ) and outer (carbonaceous, CC) Solar System materials [e.g., 1]. As Mo and $\mathrm{W}$ have very similar cosmochemical characteristics, combined W-Mo isotope systematics are of particular interest. However, a comprehensive evaluation has thus far been hampered by the scarcity of precise ${ }^{183} \mathrm{~W}$ data. To overcome this limitation, we have obtained high-precision $\mathrm{W}$ isotope data for a large set of samples for which also Mo isotope data exist, including numerous bulk (CC, NC) meteorites, acid leachates from an ordinary chondrite, as well as bulk samples and mineral separates of Type B CAIs.

In line with components and acid leachates from carbonaceous chondrites [2,3], the OC leachates show large and correlated nucleosynthetic $\mathrm{W}$ and Mo isotope anomalies that can be attributed to $s$-process variations. This demonstrates that $s$-process $\mathrm{W}$ and Mo nuclides have a common origin and are hosted in the same (presolar) carrier(s). By contrast, the investigated Type B CAI samples show no resolvable $\mathrm{W}$ isotope variations, but large and uniform Mo isotope anomalies that predominantly reflect an $r$-excess [4], advocating an independent origin of $r$-process $\mathrm{W}$ and Mo nuclides. Our new bulk meteorite data confirm the previous observation [e.g., 5] that $\mathrm{NC}$ meteorites have no resolved $\mathrm{W}$ isotope anomalies, whereas $\mathrm{CC}$ meteorites show correlated nucleosynthetic $\mathrm{W}$ and Mo anomalies. The latter are consistent with $s$-process variations, but also imply that $\mathrm{CC}$ meteorites have an overall excess of material enriched $r$ process nuclides of Mo, but not of $\mathrm{W}$ (as represented by typical Type B CAIs). Combined, these multi-scale observations provide a more comprehensive understanding of the sources, processing, and transport of material in the early Solar System, which will be discussed at the conference.

[1] Budde et al. (2019) Nature Ast. 3, 736-741. [2] Budde et al. (2016) EPSL 454, 293-303. [3] Burkhardt et al. (2012) EPSL 357-358, 298-307. [4] Burkhardt et al. (2011) EPSL 312, 390-400. [5] Worsham et al. (2019) EPSL 521, 103-112. 